\title{
Les noms abstraits intensifs et la légitimation de l'article défini
}

\author{
Delphine Beauseroy \\ ATILF-CNRS, associée au STL \\ delphine.beauseroy@atilf.fr
}

\section{Introduction}

Dans ce travail, nous nous limitons à l'étude des noms abstraits apparentés à des prédicats verbaux ou adjectivaux, et plus précisément les noms abstraits qualifiés d'intensifs par Van de Velde (1995) et Flaux \& Van de Velde (2000).

1. gentillesse, loyauté, patience, fureur, tristesse, joie, admiration, haine, jalousie, etc.

Dans un premier temps, nous verrons que ces noms présentent un fonctionnement morphosyntaxique particulier, notamment en ce qui concerne leur introduction par un article défini. En effet, nous montrerons que la présence de l'article défini en tête du syntagme nominal est nécessairement corrélée à la présence d'un élément spécifique au sein de ce syntagme.

L'analyse des types de dépendances qui légitiment l'emploi de l'article défini aux côtés des noms abstraits intensifs nous conduit alors à postuler que ces noms sont intrinsèquement relationnels (Beauseroy, 2009). Nous proposons alors une formalisation syntaxique permettant d'en rendre compte.

Enfin, nous pouvons ajouter que cette propriété ne se voit pas reflétée lorsque ces noms prennent une interprétation d'occurrence puisque leur détermination n'est plus contrainte. Dans cette seconde lecture, nous postulons que le caractère relationnel des noms n'est que facultatif.

\section{Noms abstraits intensifs et article défini}

\subsection{En emploi anaphorique}

Comme nous le montre (2), la reprise anaphorique des noms concrets est possible, d'où l'utilisation non restreinte de l'article défini à partir de la deuxième mention.

2. J'ai acheté des fruits et de la glace pour le dessert. Les fruits étaient bien rafraîchissants, mais la glace avait déjà fondu avant que je ne la serve.

Les noms abstraits intensifs se distinguent sur ce plan des noms concrets, qu'ils soient massifs, comme glace ou comptables comme fruit(s), présentés sous (2).

En effet, une propriété distinctive des noms abstraits intensifs est leur difficulté à être introduits à partir de la seconde mention par l'article défini.

3. a. Paul a eu de la patience. [...]* La patience était remarquable.

b. Il a eu de la patience. [...] \{Cette / sa\} patience était remarquable.

4. a. Pierre a montré de la fureur. [...]* La fureur était dirigée contre ses supérieurs.

b. Pierre a montré de la fureur. [...] \{Cette / sa $\}$ fureur était dirigée contre ses supérieurs.

5. a. Marie a de l'admiration pour son directeur. [...]* L'admiration est telle qu'elle ne peut lui adresser un mot sans rougir.

b. Marie a de l'admiration pour son directeur. [...] $\{$ Cette / son $\}$ admiration est telle qu'elle ne peut lui adresser un mot sans rougir. 


\subsection{En emploi cataphorique}

Toutefois, l'article défini peut également, avec les noms concrets, prendre une valeur «cataphorique » (Zribi-Hertz, 2003, qui se fonde sur Guillaume, 1975 ou Kleiber, 1990), autrement dit, recevoir une légitimation dès la première occurrence du nom par le biais d'un constituant postnominal (6) :

6. a. L'eau du lac était glacée.

b. Le chien de ma voisine est tout petit.

En (6), l'eau et le chien renvoient à des entités identifiées à l'aide du contexte qui suit le nom : l'eau à laquelle le nom renvoie est celle du lac, le chien auquel le nom renvoie est celui de ma voisine. Ils se distinguent en cela de l'exemple (2) dans lequel le nom renvoie à un élément présent dans le contexte antérieur.

Le contraste entre les exemples (2) et (3-5) révèle que l'article défini associé à un nom abstrait intensif en seconde mention est difficile. Il devient cependant possible si le nom est associé à un complément en [de SN] contenant le nom dénotant l'entité caractérisée par la propriété (7-9) :

7. a. La méchanceté [de cette remarque] était flagrante.

b. ??La méchanceté était flagrante.

c. Cette remarque était méchante.

8. a. La tristesse [de ma soeur] ne diminuait pas.

b. ??La tristesse ne diminuait pas.

c. Ma sœur était triste.

9. a. L'admiration [de Paul] pour toutes les personnes célèbres était incompréhensible.

b. ??L'admiration pour toutes les personnes célèbres était incompréhensible.

c. Paul admire les personnes célèbres.

Ces structures sont parallèles à celle présentée en (6) pour les noms concrets.

Pour les noms concrets, la présence d'une relative restrictive, au même titre que celle d'un constituant de type [de SN], impose le choix de l'article défini comme déterminant du nom tête du syntagme (10-11a).

10. a. La peinture qui servira pour le couloir est un peu trop foncée.

[vs. b. J'ai acheté de la peinture pour la chambre de Grégory, mais la peinture est un peu trop foncée.]

11. a. La table qui est dans le salon n'est plus très belle.

[vs. b. Mon frère a reçu sa nouvelle table. Mauvaise nouvelle, la table était livrée en kit !]

L'article défini peut également déterminer un nom abstrait intensif lorsque celui-ci est légitimé par une relative (12-14a), mais la présence de la relative est alors primordiale puisque nous avons vu précédemment que l'emploi anaphorique n'est pas permis :

12. a. La loyauté [que Pierre a montrée] $]_{\text {Relative }}$ était sincère.

b. *La loyauté était sincère.

13. a. La haine [que Pierre éprouvait pour son frère $]_{\text {Relative }}$ était absolue.

b. ??La haine était absolue.

14. a. La joie [qui l'a envahie à l'annonce des résultats] $]_{\text {Relative }}$ faisait plaisir à voir.

b. ??La joie faisait plaisir à voir.

Crucialement, l'acceptabilité de l'article défini dans les structures (a) de (12-14) tient au fait que la relative modifiant le nom abstrait intensif contient le SIEGE de l'entité dénotée par le nom ou un pronom, ici $l$ ', le reprenant. 
Ainsi, en (15-17), où ce nom n'est pas présent dans la relative, nous trouvons l'article un qui accompagne le nom abstrait intensif en cas de modification :

15. a. *Marie a montré la sensibilité [qui nous a attendris] $]_{\text {Relative }}$.

b. Marie a montré une sensibilité [qui nous a attendris $]_{\text {Relative. }}$.

16. a. *Ses actes envers autrui témoignent du mépris [qui fait vraiment peur] $]_{\text {Relative. }}$

b. Ses actes envers autrui témoignent d'un mépris [qui fait vraiment peur] $]_{\text {Relative. }}$

17. a. *Pierre a fait preuve de la concentration [qui était imperturbable] $]_{\text {Relative }}$.

b. Pierre a fait preuve d'une concentration [qui était imperturbable $]_{\text {Relative. }}$

Ainsi, la présence de l'article défini en tête d'un syntagme nominal qui contient un nom abstrait intensif est liée à la présence d'une construction [de SN] ou d'une relative, contenant le SIEGE de l'entité dénotée par le nom. Mis à part ces contextes, seul l'article partitif (en l'absence de modification), ou encore les déterminants démonstratifs et possessifs (en seconde occurrence) sont possibles.

18. a. Paul a eu de la patience. *La patience était remarquable.

b. Pierre a montré de la tristesse. *La tristesse était sincère.

19. a. Marie a beaucoup de charme et elle le sait. C'est pourquoi elle n'hésite pas à jouer de \{son / ce\} charme quand elle en a besoin.

b. Max a montré beaucoup de jalousie quand sa meilleure amie lui a annoncé qu'elle allait se marier. \{Sa / cette\} jalousie l'a poussé à lui avouer enfin ses sentiments.

Cependant, en contexte générique, la présence d'un article défini singulier devant les noms abstraits intensifs n'est en rien distinctive, puisque c'est l'article typique de l'emploi, indépendamment du type de nom qu'il introduit. Les noms abstraits intensifs ne se comportent donc pas différemment des autres types de noms, qu'ils soient concrets ou abstraits, massifs ou comptables :

20. a. Le lion est le roi des animaux.

b. Le sucre doit être restreint pour une bonne alimentation.

c. La passion ne dure jamais longtemps.

d. Le suicide est un acte désespéré.

Comme le note Dobrovie-Sorin (2005), la référence à l'espèce, caractéristique des phrases génériques, c'est-à-dire des phrases atemporelles conditionnées par la présence d'un syntagme nominal générique en position sujet, se fait dans les langues romanes au moyen de l'article défini.

\section{3 [le Nabs.itensif de SN] / [le Nabs.intensif Relative] : des constructions prédicatives}

Comme dans le cas des noms concrets, les constructions [de SN] et les relatives qui accompagnent le nom abstrait intensif introduit par l'article défini sont le reflet d'une prédication sous-jacente. Les exemples (21) et (23) illustrent cette observation :

21. a. La rage [que Marie a exprimée] (m’a fait peur).

b. Marie a exprimé de la rage (et cela m'a fait peur).

cf 22.a. la bière que tu as apportée

b. Tu as apporté de la bière.

23. a. (Je n'ai pas supporté) la malveillance [de ton frère].

b. Ton frère est malveillant (et je ne l'ai pas supporté).

cf 24.a. les vêtements de ma sœur 
Dans les exemples ci-dessus, le syntagme nominal complexe des exemples (a) peut être paraphrasé par une proposition temporalisée minimalement simple (b) (Zribi-Hertz, 1998). Dans le cas d'un syntagme nominal complexe avec relative, le nom abstrait intensif antécédent est intégré au sein de la relative pour créer cette paraphrase (25) :

\section{5. a. Syntagme nominal : Nabs.intensif [que SN V]}

le courage que tu as montré

b. Proposition: $\quad$ SN V Nabs.intensif

Tu as montré du courage.

Dans le cas d'un syntagme nominal avec une dépendance [de SN], le SN ainsi enchâssé est restitué dans la position sujet, le nom abstrait intensif (ou son équivalent adjectival ou verbal) dans le prédicat (26) :

26. a. Syntagme nominal : Nabs.intensif [de SN]

le courage de Max

b. Proposition : $\quad$ SN V Nabs.intensif / SN être Adj

Max a du courage. / Max est courageux.

Les syntagmes de type [le Nabs.intensif de $\mathrm{SN}$ ] sont donc comparables sur ce point aux constructions possessives (24).

Comme le montrent les exemples (21-23), les constructions [le Nabs.intensif de SN] et [le Nabs.intensif Relative] partagent avec les noms concrets en construction possessive ou relativisés le fait d'être introduits, dès la première mention, par l'article défini. Or, cela n'est pas le cas si le nom abstrait intensif n'est pas accompagné d'une dépendance :

27. a. La loyauté * (que Zoé a montrée) était sincère.

b. Nous avons été indignés par le mépris * (que Paul a pour son frère).

28. a. La méchanceté *(de Max) a été terrible.

b. La stupeur *(de Pierre) nous a surpris.

cf 29. a. J'ai démissionné de mon ancien travail car le poste ??(que je convoitais) vient de se libérer et m’a été confié.

b. J'ai acheté le nouvel album ??(de Rose).

Les exemples (27) et (28) montrent que l'article défini n'est pas acceptable en première mention si le nom n'est pas accompagné d'une dépendance [de $\mathrm{SN}$ ] ou d'une relative. L'article partitif est l'article naturellement employé lorsque le nom abstrait intensif n'est pas modifié :

30. a. Max a montré $\{$ de la $/ *$ la $\}$ méchanceté.

b. Pierre éprouve $\{\mathrm{du} / * \mathrm{le}\}$ chagrin.

Enfin, une dernière propriété renforce l'idée que la construction [le Nabs.intensif de SN] doit être analysée comme une construction possessive: [le Nabs.intensif de SN] alterne librement avec une construction [son Nabs.intensif]. Inversement, le complément ne peut être pronominalisé (Milner 1982, Godard 1986). Les exemples (31-32) illustrent cette propriété :
31. a. la singularité de Max
b. sa singularité
c. *la singularité de lui
32. a. la joie de Max 

b. sa joie
c. *la joie de lui

Nous notons en effet que le déterminant possessif est possible avec l'ensemble des noms abstraits intensifs :

33. a. Il a révélé son courage / son imprudence.

b. Le sourire qu'il arbore est révélateur de sa joie.

c. Son mépris envers les SDF fait peine à voir.

Ce déterminant possessif est bien l'équivalent du complément du nom [de X] dans [le $\mathrm{N}$ de $\mathrm{X}$ ] comme le montre le fait que le complément ne peut pas être utilisé en cooccurrence avec le possessif, puisque celuici le pronominalise.

34. a. la méchanceté de $[\text { Pierre }]_{\mathrm{i}}$

b. $[\mathrm{sa}]_{\mathrm{i}}$ méchanceté

c. ${ }^{*}[\mathrm{sa}]_{\mathrm{i}}$ méchanceté de $[\text { Pierre }]_{\mathrm{i}}$

Parallèlement à (34), le déterminant possessif est également en distribution complémentaire avec la relative restrictive :

35. a. la méchanceté que Pierre a montrée

b. sa méchanceté

c. *sa méchanceté que Pierre a montrée

\section{Les noms abstraits intensifs : des noms relationnels}

\subsection{Un argument obligatoire}

Considérons les exemples (36-37) :

36. a. $\{? ? \mathrm{La} / *$ une /*de la $\}$ méchanceté était flagrante.

b. La méchanceté [de cette remarque] était flagrante.

c. La méchanceté [dont tu as fait preuve] était flagrante.

d. Paul a fait preuve de méchanceté.

37. a. $\{? ? \mathrm{Le} / * \mathrm{un} / * \mathrm{du}\}$ découragement a été total quand il a pris conscience du travail qu'il lui restait à fournir.

b. Le découragement [de Paul] a été total quand il a pris conscience du travail qu'il lui restait à fournir.

c. Le découragement [qui a submergé Paul] fut total quand il a pris conscience du travail qu'il lui restait à fournir.

d. Paul a montré du découragement.

Crucialement, la distinction entre les exemples (a) d'une part et (b-c-d) de l'autre en (36-37) tient à la présence (ou non) du nom dénotant le SIEGE de l'entité dénotée par le nom abstrait intensif. Les exemples ci-dessus témoignent donc du fait que les noms abstraits intensifs nécessitent la présence de cet élément, puisque, si tel n'est pas le cas (36-37a), les énoncés sont inacceptables. Dans l'ensemble des exemples (bc-d), la relation qui unit le nom abstrait intensif dénotant une propriété au nom dénotant le SIEGE de cette propriété est une relation de prédication, puisque, comme nous l'avons vu, les constructions possessives et les relatives $(b-c)$ sont des constructions prédicatives internes au syntagme nominal. De plus, les 
exemples montrent que l'article défini n'est légitimé que si la relation de prédication qui unit la propriété à son SIEGE s'établit au sein même du syntagme nominal (b-c), et non au sein de la phrase (d).

En effet, considérons les exemples (38), dans lesquels le nom abstrait intensif entre dans une construction possessive à possesseur non référentiel. Pour que l'article défini soit possible, la relation prédicative doit unir le nom abstrait intensif avec le nom dénotant l'entité possédant cette propriété (39). Si tel n'est pas le cas, seul un article indéfini peut apparaître :

38. a. Zoé a montré $\{$ une/*la\} patience d'ange.

b. Pierre a $\{u n / * l e\}$ courage de lion.

(vs. Pierre a le courage d'un lion.)

39. a. La suspicion de Pierre était totalement fondée.

b. J'ai été troublée par la sensibilité de Max.

Dans les exemples (38), le SIEGE de la propriété exprimée par le nom abstrait intensif n'apparaît pas dans la dépendance du nom. Le «possesseur » est alors non référentiel et l'article défini est exclu : le syntagme est nécessairement introduit par un. Les exemples (39) montrent en revanche que, sous réserve que le nom dénotant l'entité caractérisée par la propriété soit présent à l'intérieur de la construction prédicative, la construction possessive légitime la présence de l'article défini. L'explication en est simple : le nom dénotant le SIEGE de la propriété est nécessairement référentiel, puisque c'est à travers lui que le nom abstrait intensif acquiert sa référence spécifique.

Considérons à présent le cas où les noms abstraits intensifs sont accompagnés d'une relative. Comme pour les constructions possessives, seules les relatives comportant le syntagme nominal dénotant l'entité à laquelle s'applique la propriété exprimée par le nom abstrait intensif autorisent l'emploi de l'article défini :

40. a. Paul a montré une combativité [qui a fait l'admiration de tous].

b. Marie a fait preuve d'un intérêt [qui nous a impressionnés].

41. a. La combativité [que Paul a montrée] était admirable.

b. L'intérêt [dont Marie a fait preuve] était impressionnant.

Nous postulons que les relatives des exemples (40-41) ci-dessus ont le même rôle et les mêmes effets que les constructions possessives examinées en (38-39). En effet, nous pouvons remarquer que les exemples (40) présentent deux contrastes par rapport aux exemples (41). D'une part, leurs relatives ne contiennent pas l'argument du nom abstrait intensif, c'est-à-dire le SIEGE de la propriété. D'autre part, les noms abstraits intensifs y sont introduits par l'article indéfini. Ces deux propriétés sont en fait liées. Lorsque le nom dénotant le SIEGE de la propriété est présent dans la relative, ce dernier sert de support à la référence du nom abstrait intensif; de ce fait, la relative est restrictive. En revanche, si tel n'est pas le cas, la propriété dénotée par le nom abstrait intensif n'est pas identifiée; la relative est purement descriptive. Nous proposons que ces constituants soient analysés de manière identique à la modification adjectivale qui impose l'article $u n$ en tête de syntagme. L'alternance souvent possible avec un adjectif vient appuyer cette idée :
42. a. une patience d'ange
b. une patience angélique
43. a. une admiration sincère
b. une admiration qui est sincère

Nous notons cependant que l'argument du nom abstrait intensif est bien présent dans les structures (40) même s'il ne se trouve pas au sein de la relative : il apparaît comme sujet du prédicat principal. La différence observée entre (40) et (41) trouve ici son explication : seules les relatives restrictives légitiment l'emploi de l'article défini en tête de structure. 
Le choix de l'article défini n'est donc pas une caractéristique de l'emploi des noms abstraits intensifs. Comme pour les autres types de noms, la présence d'une construction possessive à "possesseur " référentiel ou la présence d'une relative restrictive imposent l'utilisation de l'article défini en tête du syntagme nominal dans lequel elles sont enchâssées (Zribi-Hertz, 2003, Knittel, 2009).

\subsection{Hypothèse}

Pour rendre compte du comportement de l'article défini vis-à-vis des noms abstraits intensifs, nous avançons l'hypothèse que ces noms sont intrinsèquement relationnels, au même titre que frère, oncle, etc (Beauseroy, 2009). Plus spécifiquement, nous postulons que les noms abstraits intensifs nécessitent un argument, qui peut être réalisé comme un "possesseur ». Ils se distinguent en cela des autres types de noms (garçon, livre, maison, chien, etc.), qui, eux, ne nécessitent pas d'argument (Partee, 2000, Heller, 2002), et pour lesquels la construction possessive n'est que facultative. La présence d'un article défini étant une propriété liée à l'emploi de la construction possessive, l'hypothèse proposée permet d'expliquer les particularités distributionnelles de l'article défini avec les noms abstraits intensifs.

Les noms relationnels sont des noms qui, par définition, impliquent une référence à un autre terme. Les exemples (44) en sont représentatifs :

44. a. Tu ne connais pas le mari de Zoé ?

b. La collègue de Jean m'a offert des fleurs.

En (44), mari et collègue sont tous deux des noms relationnels. L'identification de l'individu dénoté par mari ne peut se faire que par le rapport que le référent du nom entretient avec une autre entité, ici Zoé. Il en est de même en (44b) où le nom collègue nécessite la présence d'un autre référent, Jean dans notre exemple, pour que son référent soit identifié.

Le choix de l'article et la nature de la dépendance sont liés : seule la présence de l'argument du nom relationnel dans le syntagme nominal rend possible l'article défini en tête de structure (44). Ce résultat est effectivement attendu puisque les noms relationnels sont des noms dont l'argument est obligatoire. Si leur argument apparaît hors du syntagme nominal, ils sont nécessairement indéfinis :

45. Marie a $\left\{u n /{ }^{*} l e\right\}$ cousin.

De plus, en l'absence de ce rapport avec une autre entité, un nom relationnel ne peut acquérir de référence, d'où le problème d'acceptabilité des exemples suivants :

46. a. ??J'ai enfin appelé $\{$ la / une $\}$ mère.

b. ??J'attends $\{1 \mathrm{a} /$ une $\}$ sœur.

Les propriétés des noms abstraits intensifs que nous avons pu observer sont ainsi parallèles à celles des noms relationnels.

Les travaux de Partee (2000) distinguent deux types de noms : d'une part, les noms qui présentent la particularité de nécessiter un argument au même titre que les verbes (les nominalisations événementielles complexes (CEN : Grimshaw, 1990) et les noms relationnels), et d'autre part, les noms qui ne nécessitent pas cet argument. Ces derniers peuvent toutefois entrer dans une construction possessive facultative. Partee montre que la relation sémantique qui unit le possesseur et le possédé est alors libre, n'est pas restreinte à la relation d'appartenance, de possession au sens strict ou de contrôle :

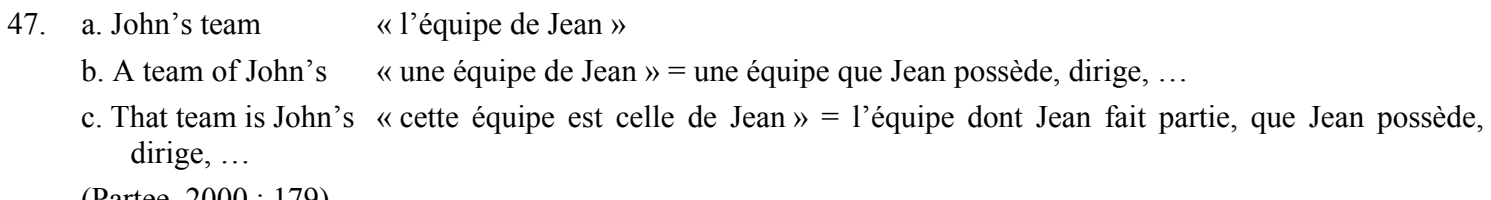

(Partee, 2000 : 179) 
À travers cet exemple, Partee montre que l'interprétation de la construction possessive n'est pas imposée, puisque, en (47), la relation sera déterminée par le contexte.

Les travaux de Heller (2002) et Vikner \& Jensen (2002) vont dans le même sens. Selon Heller, une position argumentale qui représente la relation génitive est accessible dans la structure lexicale des noms relationnels. Pour les autres, cette position argumentale est créée à l'aide d'une règle lexicale. Ainsi, lorsque le nom n'est pas relationnel, la présence de la construction possessive permet de le transformer en un tel nom en insérant une position argumentale dans la structure nominale. Il en résulte donc un contraste entre les noms relationnels, nécessairement employés en construction possessive, et les noms qui ne le sont pas, puisque cette construction n'est que facultative.

Ce contraste est illustré sous (48-50) :

48. a. ?? J'ai vu $\{$ un $/ 1$ ' $\}$ oncle.

b. J'ai vu l'oncle de Zoé.

49. a. (??) Nous avons rencontré $\{1 \mathrm{a} / \mathrm{une}\}$ collègue.

b. Nous avons rencontré la collègue de Max.

50. a. J'ai visité une maison.

b. J'ai visité la maison de Max.

La mauvaise acceptabilité de l'exemple (48a) tient au fait que le nom relationnel oncle n'est pas accompagné par son argument obligatoire. Si cet argument est présent, comme en (48b), il apparait sous la forme d'un possesseur, et la structure redevient plus acceptable. La situation est légèrement différente en (49a), dans la mesure où l'argument du nom collègue peut ne pas être exprimé ouvertement. La phrase sera néanmoins acceptable si cet argument est recouvrable contextuellement. Le possesseur est donc nécessaire seulement sur le plan sémantique, et non sur le plan syntaxique, d'où l'interprétation possible de (51) où des noms relationnels apparaissent dépourvus de leur argument :

51. Dans la famille Cerise, je voudrais le père et la mère.

Bien que l'argument de père et mère ne soit pas présent syntaxiquement, le contexte nous permet d'inférer que père renvoie à père de la famille Cerise, et mère à mère de la famille Cerise.

Enfin, le nom maison présenté sous (50) n'est pas intrinsèquement relationnel. Il peut donc apparaître indépendamment de la présence d'une construction possessive.

Notons que l'argument des noms abstraits intensifs peut également rester implicite, comme collègue en (49), s'il peut être recouvré à l'aide du contexte. C'est ce que montre l'exemple (52) :

52. Le blessé était dans un état grave. Nous sommes parvenus à le sauver, mais, malgré nos efforts, nous n'avons rien pu faire pour calmer la souffrance (i.e. la souffrance du blessé).

L'opposition observée entre (48) et (50) transparaît également lorsque la relation possessive s'exprime au moyen du déterminant possessif, qui pronominalise le possesseur : alors qu'il est nécessaire avec les noms relationnels, il n'est que facultatif avec les autres noms.

53. a. Mon oncle est venu me rendre visite.

b. ?? $\left\{\mathrm{Un} / \mathrm{l}^{\prime}\right\}$ oncle est venu me rendre visite.

54. a. Pierre a acheté une voiture.

b. Pierre a acheté ma voiture.

Notre hypothèse permet ainsi d'expliquer à la fois la distribution des articles définis et celle des dépendances (relatives ou possesseurs) qui caractérisent les noms abstraits intensifs. En tant que noms relationnels, ces noms nécessitent la présence d'un argument. Cet argument peut, au sein du syntagme nominal, être exprimé soit par le biais de la construction possessive, soit par la présence d'une relative 
restrictive qui l'explicite. Or, ce sont justement ces constructions qui légitiment l'apparition d'un article défini cataphorique.

\subsection{Formalisation syntaxique}

\subsubsection{Relation de prédication [Nom antécédent - Relative]}

En présence d'une relative restrictive, le comportement des noms abstraits intensifs n'est pas différent de celui des autres types de noms. Le nom est relativisé. Il existe alors une relation de prédication entre le nom abstrait intensif et le nom dénotant son SIEGE. Cette relation s'établit au sein de la relative :

55. a. Nabs.intensif $f_{i}\left[\operatorname{Pro}\right.$ Rel $_{i}$ Nsiège $\left._{j} \mathrm{~V} \mathrm{e}_{\mathrm{i}}\right]$

la gentillesse $e_{i}\left[\right.$ dont $_{i}$ il a fait preuve $\left.e_{i}\right]$

b. Nabs.intensif $\mathrm{i}_{\mathrm{i}}\left[\operatorname{Pro}_{\mathrm{Rel}} \mathrm{e}_{\mathrm{i}} \mathrm{V} \mathrm{Nsiège}_{\mathrm{j}}\right]$

la nostalgie [qui $_{i} \mathrm{e}_{\mathrm{i}}$ a envahi Max]

En (55a-b), l'article défini est qualifié de cataphorique (Zribi-Hertz, 2003 d'après Vendler, 1967 ; Vergnaud, 1985) : c'est la présence de la relative restrictive qui le légitime. De ce fait, un syntagme nominal qui contient une relative restrictive apparaît dès la première mention en cooccurrence avec l'article défini. Zribi-Hertz (2003) propose une formalisation à l'aide d'un topique nul dans le spécificateur de DP, qui permet de lier l'article défini en emploi anaphorique. Lorsque ce dernier est cataphorique, le topique n'est pas mis en relation avec un élément préidentifié : l'identification doit donc se faire de manière interne au syntagme nominal. Son hypothèse, fondée sur l'analyse de Kayne (1994), est qu' " une relative prédicative est une proposition, enchâssée au sein d'un syntagme nominal, et dont un argument est extrait ». Au sein du syntagme nominal, la relative enchâssée permet donc de légitimer l'article défini.

(55b) peut donc être formalisé de la façon suivante :

56. Représentation de la construction relative : [1e Nabs.intensif relative]

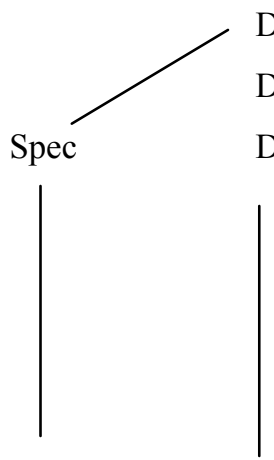

$\varnothing_{\mathrm{z}}$
$1 \mathrm{a}_{z}$

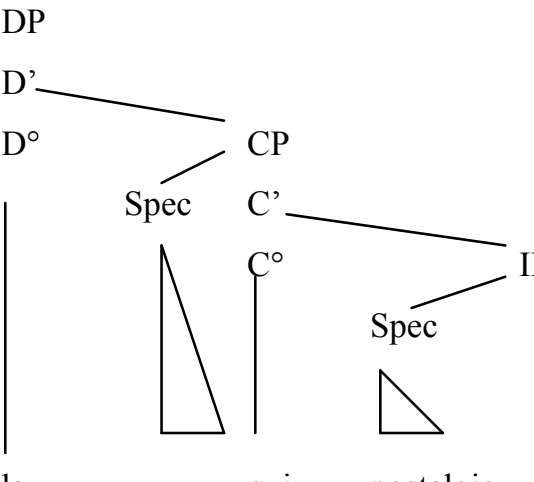

qui nostalgie

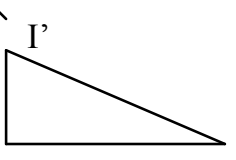

a envahi Max 


\subsubsection{Les constructions possessives}

Nous proposerons une formalisation syntaxique de la construction « possessive », qui unit le nom abstrait intensif et son argument, parallèle à celle que Knittel (2009) a proposée pour les noms simples.

Knittel $(2007,2009)$ propose une analyse qui regroupe sous la même étiquette les structures [N de $S N]$, comme livre d'un enfant, et les structures $[N$ de $N]$, comme livre d'enfant. Toutes deux sont analysées comme des constructions possessives, les premières étant caractérisées par la présence d'un possesseur référentiel, tandis que les secondes sont dotées d'un possesseur non référentiel. Knittel tente ainsi de rendre compte de façon uniforme de ces différentes constructions possessives.

Adoptant l'analyse sémantique de Heller (2002), Knittel suggère l'existence d'une projection RelP, dont la tête est $d e$, qui transforme un nom simple en un nom relationnel en permettant l'introduction d'un argument. Le «possesseur » est alors généré en spécificateur de RelP, et «l'objet possédé » en position complément :

\section{Construction possessive :}

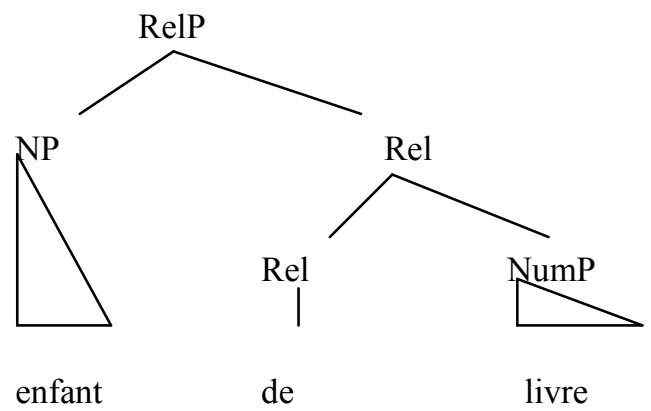

Dans le cas des constructions à possesseur référentiel, Knittel s'accorde avec l'analyse de Kayne (1993, 1994) et postule qu'une projection AgrP (dont le rôle est de vérifier le cas du possesseur) liée à la flexion possessive, est nécessaire. En effet, lorsqu'il est référentiel (c'est-à-dire [+nombre]), le possesseur a des traits à vérifier : il subit alors un mouvement vers le spécificateur d'AgrP pour la vérification du cas.

Par ailleurs, le possesseur apparaît nécessairement en français à la droite du nom tête, c'est-à dire du possédé. Knittel fait alors l'hypothèse que la structure d'une construction possessive est toujours dominée par une projection fonctionnelle FP, liée à la prédication, vers laquelle tant le possesseur que le possédé se déplacent.

La représentation (58) formalise cette hypothèse :

58. Représentation de la construction à possesseur référentiel

(Knittel, 2009)

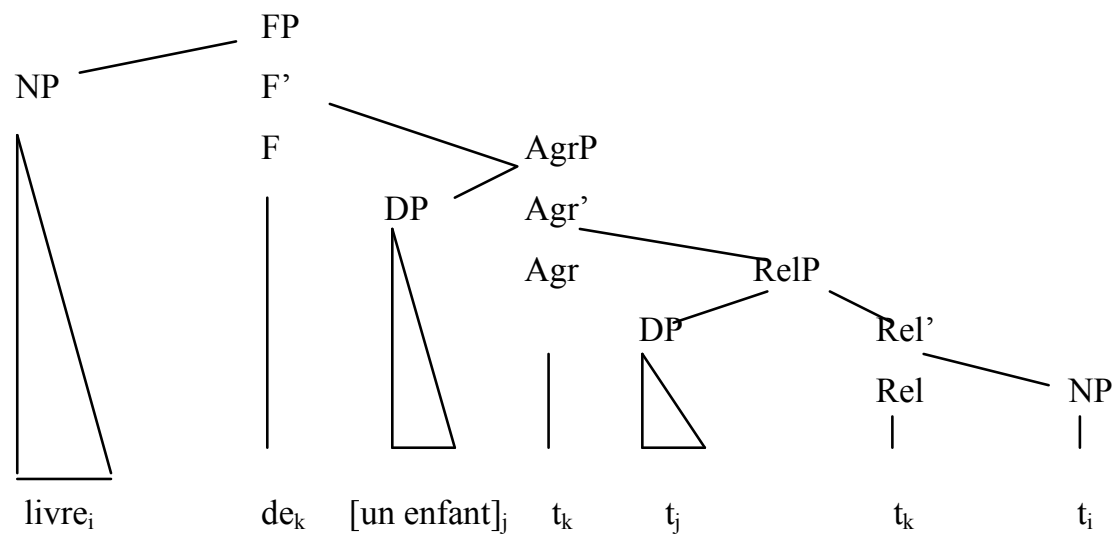


Knittel ajoute que c'est la présence de la projection AgrP qui rend nécessaire l'article défini. Pour cela, elle s'appuie sur des exemples tels (59), qui montrent qu'avec les constructions à possesseurs non référentiels, c'est l'article indéfini qui est choisi en première occurrence :

59. a. Je n'ai pas pu arriver plus tôt : [une voiture de sport] était garée devant mon garage.

(Knittel, 2007 : 166)

b. J'ai dessiné [une branche d'arbre].

En (59), sport et arbre ne sont pas référentiels. Knittel les analyse alors comme de simples NP. Suivant Longobardi (2000), pour qui les NP ne nécessitent pas de cas, Knittel suggère que la projection RelP n'est alors pas dominée par celle d'AgrP. En première mention, c'est l'article indéfini qui est nécessairement employé, (60a) et (60b) étant difficilement acceptables s'il n'a pas été fait mention de la voiture de sport ou de la branche d'arbre dans un contexte antérieur :

60. a. ??Je n'ai pas pu arriver plus tôt : [la voiture de sport] était garée devant mon garage.

b. ??J'ai dessiné [la branche d'arbre].

L'exemple (59a) s'oppose ainsi à (61), où des voisins est référentiel et où l'article défini est obligatoire :

61. a. Je n'ai pas pu arriver plus tôt : [la voiture des voisins] était garée devant mon garage.

vs. b. ??Je n'ai pas pu arriver plus tôt : [une voiture des voisins] était garée devant mon garage. (acceptable en lecture partitive seulement)

Puisqu'un possesseur référentiel nécessite la présence de la projection AgrP et que AgrP entraîne l'article défini (cf. 61a vs. 61b), nous pouvons en conclure que l'article défini est l'article caractéristique des constructions possessives à possesseur référentiel.

Knittel $(2009,2010)$ étend cette analyse aux noms événementiels. Pour Grimshaw (1990), les noms événementiels présentent des propriétés caractéristiques. Outre leur invariabilité en nombre, ils nécessitent un argument, au même titre que les verbes auxquels ils sont apparentés. Grimshaw identifie cet argument comme le complément du verbe correspondant :

62. a. La construction de cette maison a duré 6 mois.

b. On a construit cette maison en 6 mois.

c. ?? La construction a duré 6 mois. (acceptable si l'objet est sous-entendu)

63. a. La consommation d'alcool est interdite.

b. Il est interdit de consommer de l'alcool.

c. ?? La consommation est interdite.

De plus, une troisième propriété syntaxique relevée par Grimshaw tient à la nécessité de l'article défini en tête de SN, comme en témoignent (64) :

64. a. *Une construction de la maison a duré six mois.

b. *Une consommation d'alcool (par les mineurs) est interdite.

Ainsi, à la suite de son analyse, Knittel rend compte du fait que les propriétés mises en évidence par Grimshaw (1990) ne sont pas des propriétés caractéristiques des noms événementiels, mais des propriétés caractéristiques des constructions possessives dans lesquelles ces noms sont employés.

Nous proposons alors la formalisation (65) pour rendre compte des constructions possessives qui unissent le nom abstrait intensif et son SIEGE : 


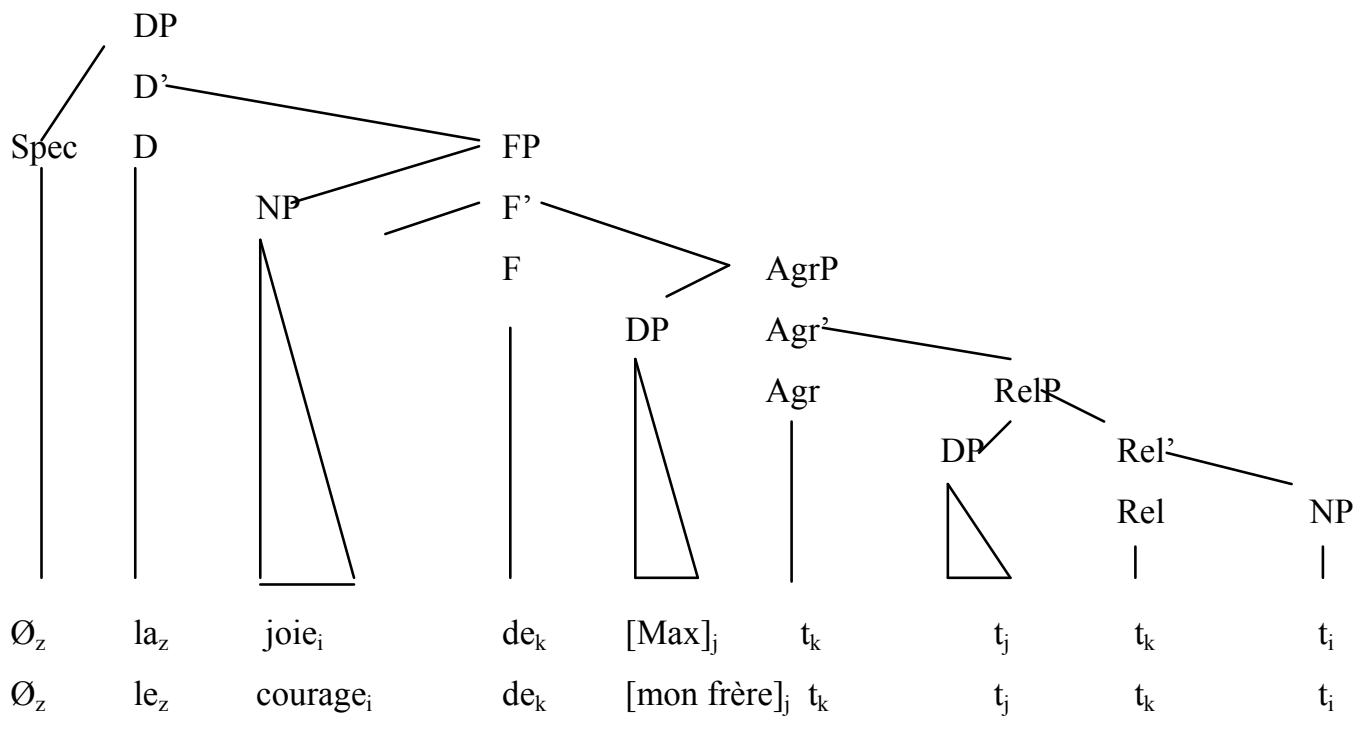

En syntaxe, rien ne différencie les noms abstraits intensifs des autres, si ce n'est le caractère obligatoire du SIEGE.

\subsubsection{Synthèse}

L'étude des syntagmes nominaux présentant des constructions possessives et des relatives nous indique que l'article défini utilisé pour introduire ces syntagmes est dépendant de la relation de prédication. En effet, tous peuvent être paraphrasés par une proposition finie indépendante, dans laquelle le nom tête du syntagme complexe d'origine apparaît dans un syntagme nominal simple, et dans laquelle il est introduit par un déterminant indéfini (article indéfini ou partitif). Comme l'ont souligné de nombreux auteurs (Milner, 1982 ; Godard, 1986 ; Zribi-Hertz, 2003, entre autres), l'article défini n'est donc pas l'article du nom antécédent ou l'article du nom tête. Cette constatation conduit à analyser les syntagmes nominaux déplacés dans ces structures prédicatives comme étant à l'origine des syntagmes non déterminés, c'est-àdire des NumP (si le nom est comptable) ou des NP (si le nom est massif).

\subsection{Quelques remarques complémentaires}

Nous avons pu conclure que, hors interprétation générique, l'article défini est utilisé avec les noms abstraits intensifs dans un emploi cataphorique, puisqu'il est dépendant de la présence d'une expansion à la droite du nom. Cependant, à la suite de l'analyse présentée ci-dessus, il nous faut alors rendre compte des exemples (66-67), qui, bien que moins habituels, présentent l'article défini au côté de noms abstraits intensifs, sans que le nom ne soit suivi d'une expansion :

66. a. Le conducteur n'a pas pu lutter contre la fatigue et s'est endormi au volant.

b. À neuf reprises, il s'y est fracturé le nez dans le paquet des avants. Se jeter dans la mêlée, il sait faire. Mais la combativité sera insuffisante pour boucler [...] les grandes lignes de l'Uruguay Round.

67. a. Le blessé était dans un état grave. Nous sommes parvenus à le sauver, mais, malgré nos efforts, nous n'avons rien pu faire pour calmer la souffrance.

b. Max avait tout planifié minutieusement pour sa demande en mariage. Sa fiancée ne se doutait de rien, l'étonnement était total. 
Dans les exemples (66-67), les noms abstraits intensifs souffrance, combativité, fatigue et étonnement n'entrent pas dans des constructions [le N de N] ou [le N Relative]. Pourtant, la présence d'un article défini ne conduit pas à l'inacceptabilité de ces exemples.

En (66), les syntagmes la fatigue et la combativité sont en fait des syntagmes nominaux génériques et renvoient respectivement aux propriétés génériques, aux concepts, de fatigue et de combativité. En effet, la fatigue (66a) ne renvoie pas à la fatigue du conducteur, mais bien à la fatigue « en général », comme en témoigne l'inacceptabilité de (68) :

68. ??Le conducteur n'a pas pu lutter contre sa fatigue et s'est endormi au volant.

L'emploi de l'article défini en (66) n'est pas contradictoire avec les hypothèses que nous avons avancées puisque l'emploi générique autorise tout type de nom à être introduit par $l e$.

Considérons maintenant les exemples présentés sous (67) qui semblent plus problématiques. En effet, le nom abstrait intensif n'est plus dans un emploi générique, comme le montre l'alternance possible entre l'article défini et le déterminant possessif :

69. a. Le blessé était dans un état grave. Nous sommes parvenus à le sauver, mais, malgré nos efforts, nous n'avons rien pu faire pour calmer $\{\mathrm{la} / \mathrm{sa}\}$ souffrance.

b. Max avait tout planifié minutieusement pour sa demande en mariage. Sa fiancée ne se doutait de rien, \{l' / son\} 'étonnement était total.

Puisque aucun élément explicite conjoint au nom abstrait intensif ne peut légitimer l'article défini, comment rendre compte de ces exemples?

Kleiber (1999) tente d'expliquer ce phénomène. Selon lui, il s'agit là d'anaphores associatives, c'est-àdire d'anaphores qui permettent d'introduire un nouveau référent grâce au lien qu'il entretient avec le référent d'un élément déjà introduit :

70. Il s'approcha d'une maison toute neuve. Les murs / le toit / les fenêtres étai(en)t fait(e)s en aluminium.

(Kleiber, $1999: 73$ )

En (70), le référent de l'expression anaphorique les murs, le toit ou les fenêtres est interprété par le biais du référent déjà introduit par maison. Pour qu'un tel lien puisse s'établir, le rapport entre les deux référents doit être pertinent. Or une maison a nécessairement des murs, un toit, etc., d'où l'acceptabilité de (70).

Pour Kleiber, pour qu'une anaphore associative soit possible, deux conditions doivent être remplies : la condition d'aliénation et le principe de congruence ontologique.

Or, les noms abstraits intensifs sont en opposition avec ces deux principes : d'une part, ils ne peuvent être perçus de façon autonome, et d'autre part, alors que l'entité dénotée par le nom abstrait intensif ne possède pas de matière et de forme propre, son référent en possède. L'anaphore associative, si c'est de cela qu'il s'agit en (69), ne devrait donc pas être autorisée dans les exemples ci-dessus.

Une analyse possible serait de considérer souffrance et étonnement comme présentant, dans ces exemples, une ellipse de la construction possessive. 


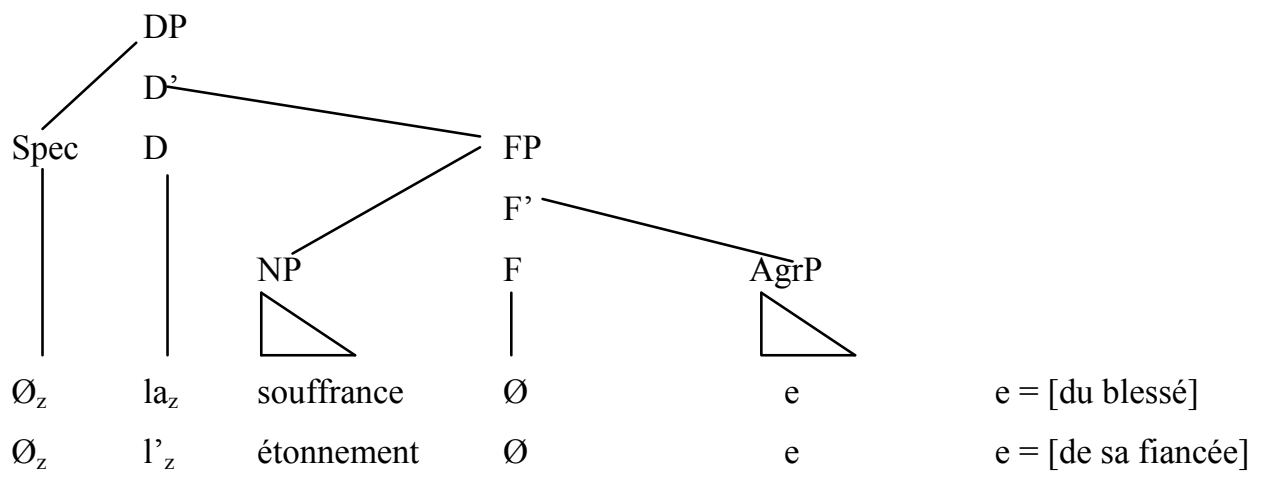

\section{En lecture d'occurrence, une détermination moins restreinte}

Les noms abstraits intensifs ont pour la plupart une seconde lecture, dans laquelle ils dénotent des occurrences (manifestations particulières de l'entité dénotée par le nom abstrait intensif). Ces occurrences peuvent être abstraites $(72 a)$ ou concrètes $(72 b)$ :
72. a. Paul a commis une imprudence.
$=$ acte d'imprudence
b. Pierre a ôté les saletés qui étaient sur la table.
$=$ choses sales

Le comportement des noms de propriété en lecture d'occurrence est comparable à celui des noms comptables :

73. a. $\{$ Une / l'\} imprudence a conduit hier à un terrible carambolage.

b. J'ai \{une / la\} rougeur sur le bras.

Les exemples (73) sont tout à fait acceptables. Les noms imprudence et rougeur sont ici dans leur acception de nom dénombrable. L'incompatibilité de l'article indéfini avec ces noms n'a donc plus lieu d'être.

Le comportement des noms abstraits intensifs en lecture d'occurrence contraste avec le comportement de ces noms dans leur lecture intensive. En effet, les noms sont alors dans un emploi comptable et présentent les mêmes variations distributionnelles que celles des noms comptables concrets. En première occurrence, ils sont ainsi préférentiellement introduits par l'article indéfini.

74. a. J'ai commis une imprudence.

b. Il a prévu des douceurs pour la fin du repas.

Cependant, comme dans leur emploi intensif, l'article défini peut être légitimé dès la première mention par des constructions possessives (75), qui sont en distribution complémentaire avec le déterminant possessif, ou des relatives (76).

75. a. Les infidélités de Paul me rongent.

$=$ Ses infidélités $(*$ de Paul) me rongent.

b. Admire les beautés du paysage !

$=$ Admire ses beautés $(*$ du paysage) !

76. a. Les méchancetés que tu as proférées m'ont sincèrement blessé.

b. Un psychologue peut t'aider à gérer les peurs qui t'envahissent la nuit. 
Cependant, les noms abstraits intensifs en emploi occurrentiel se distinguent alors de leur contrepartie intensive par le fait que la présence de ces constructions (relatives ou constructions possessives) n'est pas le seul contexte autorisant l'article défini. En effet, celui-ci peut également avoir une valeur anaphorique, comme en (77) :

77. a. Paul a repéré une saleté. [...] Une seconde plus tard, la saleté avait déjà disparu.

b. En pleine réunion de famille, Zoé a piqué une colère. [...] Heureusement, la colère a vite été oubliée.

Les exemples (77) montrent que l'article défini n'est pas restreint à la présence d'une quelconque dépendance postnominale. Nous pouvons en déduire qu'en lecture d'occurrence, le nom ne possède pas d'argument obligatoire: contrairement à sa contrepartie intensive, il ne s'agit donc pas d'un nom relationnel.

\section{Conclusion}

L'étude de la détermination, et plus particulièrement des conditions d'apparition de l'article défini, nous a permis de montrer que les noms abstraits intensifs sont des noms relationnels. En tant que tels, ils nécessitent un argument, qui correspond en l'occurrence au SIEGE de l'entité dénotée par le nom, avec lequel ils entrent dans une relation de prédication au niveau syntaxique. Cette relation peut s'établir soit par le biais d'un verbe support, auquel cas le nom abstrait intensif est introduit par un article indéfini (partitif ou un s'il est modifié), soit par celui d'une construction possessive ou d'une relative au sein même du syntagme nominal dont il constitue la tête, auquel cas le nom abstrait intensif est introduit par l'article défini.

Dans le cas de la construction possessive et de la relative, c'est la présence de cette relation de prédication interne au syntagme nominal qui est responsable de la présence de l'article défini. Ainsi, dans le cas où l'argument est absent de la relative, seul l'article un est possible. Notre analyse permet donc de rendre compte du lien régulièrement observable entre la distribution des articles et celle des dépendances nominales et de leur contenu.

Ce comportement contraste avec celui de ces mêmes noms en lecture d'occurrence puisque la libre distribution des articles indique qu'ils ne nécessitent pas la présence d'un argument. Dans cette acception d'occurrence, ces noms ne sont donc pas intrinsèquement relationnels : la construction possessive n'est alors que facultative.

\section{Références bibliographiques}

Beauseroy, D. (2009). Syntaxe et sémantique des noms abstraits statifs : des propriétés verbales ou adjectivales aux propriétés nominales. Thèse de Doctorat, Université Nancy 2.

Dobrovie-Sorin, C. (2005). Généricité et détermination nominale. In C. Dobrovie-Sorin (ed.) Noms nus et généricité, Saint-Denis : Presses Universitaires de Vincennes, 5-29.

Flaux, N. \& Van de Velde, D. (2000). Les noms en français : esquisse de classement. Les essentiels du français, Paris : Ophrys.

Godard, D. (1986). Les déterminants possessifs et les compléments de nom. Langue Française, 72, 102-122.

Grimshaw, J. (1990). Argument Structure. Cambridge : MIT Press.

Guillaume, G. (1975). Le problème de l'article et sa solution dans la langue française. Québec : Presses de l'Université Laval. (éd. orig. Paris : Nizet. 1919).

Heller, D. (2002). Possession as a lexical relation : evidence from the Hebrew Construct State. In L. Mikkelsen et C. Potts (eds.) WCCFL 21 Proceedings, Somerville : Cascadilla Press, 127-140.

Kayne, R. S. (1993). Towards a modulary theory of auxiliary selection. Studia Linguistica 47-1, 3-31. 
Kayne, R. S. (1994). The Antisymetry of Syntax, Cambridge (Mass) : MIT Press.

Kleiber, G. (1990). Article défini et démonstratif. In G. Kleiber \& J-E. Tyvaert (eds.) L'anaphore et ses domaines, Recherches linguistiques XIV, Université de Metz, 199-227.

Kleiber, G. (1999). Anaphore associative et relation partie-tout : condition d'aliénation et principe de congruence ontologique. Langue française 122-1, 70-100.

Knittel, ML. (2007). Catégories fonctionnelles et déficience : étude typologique de quelques constructions verbales et nominales. Mémoire d'HdR, Université Nancy 2.

Knittel, ML. (2009). Le statut des Compléments du Nom en [de NP]. Revue Canadienne de Linguistique, 54-2, 10011032.

Knittel, ML. (2010). Possession vs. pseudo-incorporation in the nominal domain : Evidence from French event nominals dependencies. The Linguistic Review 27 (sous presse).

Longobardi, G. (2000). The structure of DPs : some principles, parameters and problems. In Handbook of Syntactic Theory, M. Baltin \& C. Collins (eds.), London : Blackwell, 562-603.

Milner, J-C. (1982). Ordres et raisons de langue. Paris : Seuil.

Partee, B. H. (2000). Genitives, Relational Nouns and the Argument-Modifier Distinction. ZAS Papers in Linguistics, $17,177-201$.

Van de Velde, D. (1995). Le spectre nominal : des noms de matières aux noms d'abstractions. Paris : Peeters.

Vendler, Z. (1967). Verbs and times. In Linguistics in Philosophy : 97-121. Ithaca : Cornell University Press.

Vergnaud, J-R. (1985). Dépendances et niveaux de représentation en syntaxe. Amsterdam \& Philadelphie : John Benjamins.

Vikner, C. \& Jensen, P.A. (2002). A semantic analysis of the English genitive. Interaction of lexical and formal semantics. Studia Linguistica, 56-2, 191-226.

Zribi-Hertz, A. (1998). Les syntagmes nominaux possessifs en français moderne : syntaxe et morphologie. In J.Guéron et A.Zribi-Hertz (éds) La grammaire de la Possession, 129-166, Nanterre : Publidix.

Zribi-Hertz, A. (2003). La syntaxe de l'article défini : de la structure à l'interprétation. Communication présentée lors du Colloque Modèles Syntaxiques, Bruxelles, 6-8 mars 2003. 